\title{
Fosfomycin Causes Transient Lysis in Escherichia coli Strains Carrying Fosfomycin-resistance Plasmids
}

\author{
By JAVIER LEÓN, JUAN M. GARCÍA-LOBO*, JESÚS NAVAS AND \\ JOSÉ M. ORTIZ \\ Departamento de Bioquimica, Facultad de Medicina, 39011 Santander, Spain
}

(Received 12 April 1985; revised 25 June 1985)

\begin{abstract}
Escherichia coli cells carrying fosfomycin-resistance plasmids show high levels of resistance towards this drug. However, the plasmid-carrying strains exhibited a transient lytic phase induced by fosfomycin when grown in rich liquid media. This lytic phase was not observed if the cells were grown in liquid minimal media. Fosfomycin-induced lysis depended on the accumulation of drug inside the bacteria, presumably as a result of the saturation of the fosfomycin modification system. Growth recovery after lysis was not due to drug inactivation in the culture medium and could be explained by selection of mutants showing impaired fosfomycin transport when high concentrations of fosfomycin were used. However, there was no selection of mutants with low drug concentrations.
\end{abstract}

\section{INTRODUCTION}

Fosfomycin (L-cis-1,2-epoxipropylphosphonic acid) is a bactericidal antibiotic which inhibits the enzyme phosphoenolpyruvate:UDP- $N$-acetylglucosamine enolpyruvyltransferase (EC 2.5.1.7) (Kahan et al., 1974). This enzyme catalyses the first step of the murein biosynthetic pathway and is found in the cytoplasm of the cell. Thus, for the antibiotic to have an effect it must enter the cell. This is mainly accomplished through the $g l p T$ transport system, which is normally used to transport $s n$-glycerol 3-phosphate (G3P) (Lin, 1976).

Spontaneous mutants of Escherichia coli resistant to fosfomycin are easily isolated. Most of these are transport-defective mutants. They cannot grow on minimal media with G3P as the only carbon source and usually carry mutations in the $g l p T$ gene (Tsuruoka \& Yamada, 1975). Mutations in other loci such as $g l p R$, cya/crp (Alper \& Ames, 1978) and ptsI (Cordaro et al., 1976) are also found among these mutants. In addition to these chromosomal mutations, plasmids encoding resistance to fosfomycin have been found. These plasmids were originally found in clinical isolates of Serratia marcescens (Mendoza et al., 1980) and have been partially characterized. We have shown that the determinant of resistance lies on a transposable element called Tn2921 (García-Lobo \& Ortiz, 1982) and the resistance gene from this transposon has been cloned and characterized (García-Lobo et al., 1984).

Plasmid-mediated resistance to fosfomycin is not due to the lack of transport of the antibiotic (León et al., 1982). We have recently reported that $E$. coli cells carrying such plasmids modify fosfomycin intracellularly to another, inactive compound (León et al., 1985). Even though the resistance of chromosomal mutants is much less than that of plasmid-carrying strains, fosfomycin can cause transitory lysis in cultures of plasmid-bearing bacteria. Here we report this feature and discuss it in connection with the mechanism of plasmid-mediated resistance to fosfomycin. 


\section{METHODS}

Bacterial strains, plasmids and culture media. E. coli 185 (resistant to nalidixic acid) and E. coli W3110 (resistant to rifampicin) are $E$. coli $\mathrm{K} 12$ derivatives susceptible to fosfomycin. $E$. coli $185 \mathrm{GP}$ is a spontaneous mutant showing diminished fosfomycin uptake (León et al., 1982). Micrococcus varians M11 is a fosfomycin-hypersensitive strain (León, 1983). The plasmid pOU900 is one of the clinically isolated fosfomycin-resistance plasmids (Mendoza et al., 1980). pSU912 is a derivative of pColE1 containing Tn2921 (García-Lobo \& Ortiz, 1982). pSU902 was obtained by cloning the fosfomycin-resistance gene into pBR322 (García-Lobo et al., 1984). The media used for lysis experiments were L broth (Davis et al., 1980), antibiotic medium no. 3 (Difco), Garen-Levinthal minimal medium, 0.3 mM-phosphate (Garen \& Levinthal, 1960) and M9 minimal medium (Davis et al., 1980). $\left[1,2-{ }^{3} \mathrm{H}\right]$ Fosfomycin (specific activity $5.3 \times 10^{8} \mathrm{~Bq} \mathrm{mmol}^{-1}$ ) was kindly supplied by Compañia Española de la Penicilina y Antibióticos (CEPA).

Lysis experiments. In a typical experiment, $3 \mathrm{ml} \mathrm{L}$ broth was inoculated with $60 \mu \mathrm{l}$ of an overnight culture, and incubated with shaking at $37^{\circ} \mathrm{C}$, unless otherwise stated. Growth was monitored by recording the $\mathrm{OD}_{600}$ in a Bausch and Lomb Spectronic 20 spectrophotometer. Different concentrations of fosfomycin were added at $\mathrm{OD}_{600}=0 \cdot 15-0 \cdot 20$ and lysis was measured by decrease in $\mathrm{OD}_{600}$.

Antibiotic susceptibility determinations. Minimal inhibitory concentrations (MICs) were determined by inoculating about $5 \times 10^{4}$ bacteria on Mueller-Hinton agar plates containing fosfomycin at concentations ranging from $2 \mu \mathrm{g} \mathrm{ml} \mathrm{m}^{-1}$ to $80 \mathrm{mg} \mathrm{ml}^{-1}$. Positive growth was recorded when more than five colonies appeared after incubation for $24 \mathrm{~h}$ at $37^{\circ} \mathrm{C}$.

Measurement of intracellular concentrations of fosfomycin. Cells incubated in the presence of $\left[{ }^{3} \mathrm{H}\right]$ fosfomycin were harvested and ultrasonically disrupted. The modified fosfomycin present in the ultrasonic extract was detected and measured in paper chromatograms as previously described (León et al., 1985).

Measurement of fosfomycin in culture broths. After incubation in liquid media containing fosfomycin, the cells were removed by centrifugation and the concentration of fosfomycin in the broths were determined by microbiological assay on Mueller-Hinton agar plates using $M$. varians M11 as indicator strain.

Characterization of mutants. The $\mathrm{G}^{3} \mathrm{P}^{-}$phenotype was detected by replicating colonies on $\mathrm{M} 9$ agar plates supplemented with $20 \mathrm{mM}-\mathrm{G} 3 \mathrm{P}$ as the only carbon source (Ludtke et al., 1982). Glycerol ${ }^{-}, \mathrm{maltose}^{-}$and fructose ${ }^{-}$ phenotypes were scored by the production of red colour in McConkey agar plates supplemented with $1 \%$ (w/v) of the mentioned carbon sources.

\section{RESULTS}

\section{Transient lysis mediated by fosfomycin}

The fosfomycin MICs determined on Mueller-Hinton agar as described in Methods were $60 \mathrm{mg} \mathrm{ml}^{-1}$ for both $E$. coli 185 (pOU900) and E. coli C600(pOU900). Similar values were found for E. coli C600 derivatives carrying the plasmids pSU902 or pSU912. The same value was obtained when the MIC was determined on L agar or in Mueller-Hinton or L broth. However, when exponentially growing cultures of $E$. coli 185 containing any of the aforementioned fosfomycin-resistance plasmids were treated with fosfomycin, they showed a transient lytic phase followed by recovery of exponential growth (Fig. 1). Transient lysis was observed in rich media such as L broth, AM3 or Mueller-Hinton broth. The extent of lysis was slightly greater in $L$ broth than in the other media used (data not shown). No lytic phase was observed when the cells were cultured in liquid minimal media, such as Garen-Levinthal medium, or in M9 supplemented with $20 \mathrm{~mm}$-glucose as the only carbon source.

Transient lysis was also observed for E. coli $\mathrm{W} 3110$ and $E$. coli $\mathrm{C} 600$ carrying any of the aforementioned plasmids. This suggested that lysis was independent of the recipient strain and of some plasmid function other than the resistance to fosfomycin. However, E. coli strain 185GP (defective in fosfomycin transport) was not transiently lysed by fosfomycin concentrations below its MIC, even if it carried a fosfomycin-resistance plasmid.

The fall in $\mathrm{OD}_{600}$ that occurred in the lytic phase was accompanied by a great loss of viability (one survivor per $10^{4}$ viable cells in L broth). These surviving cells were responsible for a second exponential growth phase which allowed recovery of the culture.

\section{Effects on the lytic phase of the concentration of fosfomycin and the growth rate}

The lytic response of E. coli 185(pOU900) after treatment with various concentrations of fosfomycin is shown in Fig. 1. Higher concentrations of fosfomycin induced earlier lytic 


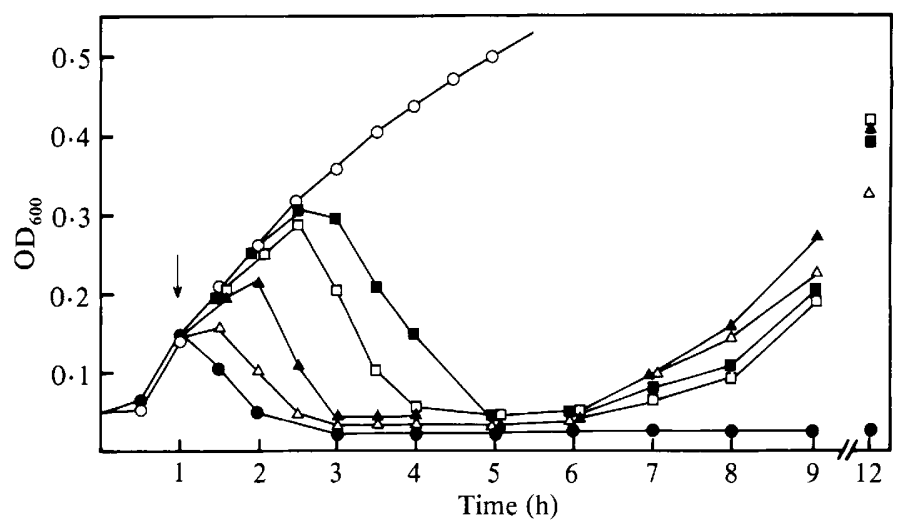

Fig. 1. Effect of the addition of different concentrations of fosfomycin to cultures of $E$. coli 185 (O) and E. coli 185(pOU900) ( $\square, \boldsymbol{\square}, \triangle, \boldsymbol{\Delta}$ ). The following concentrations of fosfomycin were used: $0.05 \mathrm{mg} \mathrm{m}^{-1}(\boldsymbol{O}, \square) ; 0.1 \mathrm{mg} \mathrm{m}^{-1}(\square) ; 1 \mathrm{mg} \mathrm{m}^{-1}(\Delta)$; and $10 \mathrm{mg} \mathrm{ml}^{-1}(\triangle)$. E. coli 185 growing without fosfomycin was included $(O)$. The cultures were inoculated at time zero. The arrow indicates the time of addition of fosfomycin.

responses. However, neither the extent of lysis nor the time of recovery was affected by the concentration of fosfomycin.

The transient lysis induced by fosfomycin depended on the growth rate of the culture. This was shown by adding fosfomycin $\left(1 \mathrm{mg} \mathrm{m}^{-1}\right.$ final concentration) to E. coli 185 (pOU900) cultures at different points during the exponential phase. Comparison of the resulting lytic periods showed that the addition of fosfomycin at the beginning of the exponential phase induced a lytic period which started later and that was shorter than that produced by the addition of fosfomycin in the middle or the end of the exponential phase.

Further evidence of the relationship between the growth rate and the characteristics of the lytic phase was obtained when the lytic periods induced by fosfomycin in cultures grown at 30 and $37^{\circ} \mathrm{C}$ were compared. Lysis onset was delayed by about $3 \mathrm{~h}$ in the slower-growing culture $\left(30^{\circ} \mathrm{C}\right)$.

\section{Detection of fosfomycin and its inactive derivative during the growth cycle}

The data shown in Fig. 1 suggested that lysis could be caused by intracellular fosfomycin, since higher fosfomycin concentrations resulted in earlier onset of lysis. Soluble extracts were prepared, as described in Methods, from E. coli 185(pOU900) cells grown in the presence of $\left[{ }^{3} \mathrm{H}\right]$ fosfomycin. Samples were taken at different points during growth to examine the amount and distribution of antibiotic inside the cells. Soon after the addition of fosfomycin, the cells contained only the inactive fosfomycin derivative. The same was true for recovered cells (second exponential phase). However, it can be seen that there was accumulation of unmodified fosfomycin in the cells before the start of the lysis (Fig. 2).

When the experiment described in Fig. 2 was done using E. coli 185(pOU900) cells grown in Garen-Levinthal minimal medium, we could not detect unmodified fosfomycin in the cells (data not shown). This result is consistent with the absence of the lytic phase in cultures of plasmid-containing strains growing in minimal media.

\section{Characterization of the recovered cells}

Recovery of exponential growth after lysis was not due to the inactivation of the antibiotic in the medium. This was demonstrated by measuring the concentration of fosfomycin in the medium of $E$. coli 185 (pOU900) cultures which had recovered from lysis produced by the addition of $0 \cdot 1$ and $1 \mathrm{mg}$ fosfomycin $\mathrm{ml}^{-1}$. Fosfomycin concentrations were determined microbiologically, as described in Methods. In every experiment, $90-95 \%$ of the initial amount of fosfomycin was present at the end of the stationary phase. 


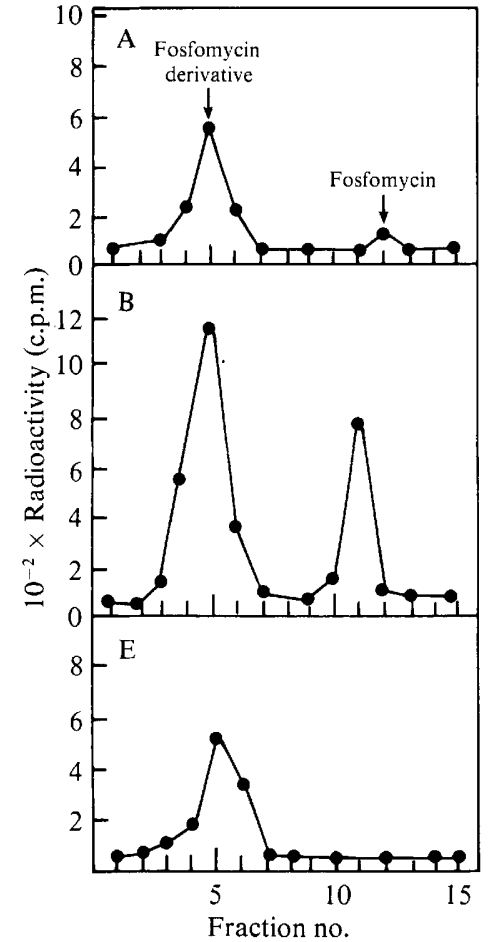

Fig. 2

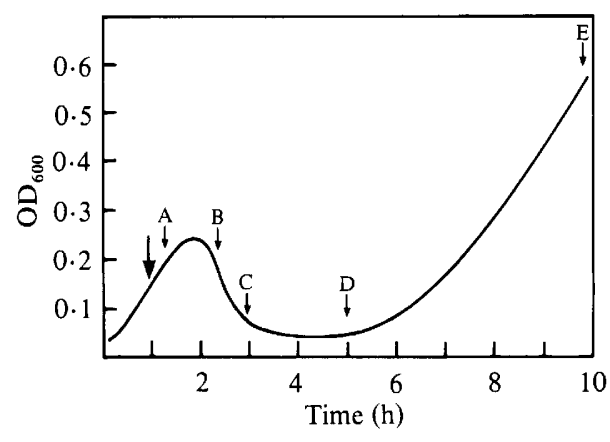

Fig. 3

Fig. 2. Paper chromatographic analysis for the presence of fosfomycin or its inactive derivatives in $E$. coli 185(pOU900) cells taken at times A, B and E (see Fig. 3). $\left[{ }^{3} \mathrm{H}\right]$ Fosfomycin was added to exponential cultures to a final activity of $3 \times 10^{5} \mathrm{c} . \mathrm{p} . \mathrm{m} . \mathrm{ml}^{-1}$. The samples applied to the chomatograms were extracts of $0.1 \mathrm{ml}$ of culture.

Fig. 3. Schematic representation of the time course of the growth of E.coli 185(pOU900) in the presence of fosfomycin. The large arrow indicates the time of addition of fosfomycin, the letters A, B, C, D and E mark times that are referred to in Fig. 2 and Table 1.

\section{Table 1. Occurrence of G3P- mutants among E. coli cells treated with fosfomycin}

The results are means of three independent experiments.

Fosfom
conen (
1
1
1
1
$0 \cdot 1$
$0 \cdot 1$

Sample
A
C
D
E
A
E

Viable count (c.f.u. $\mathrm{ml}^{-1}$ )

$$
\begin{array}{r}
6 \times 10^{7} \\
4 \times 10^{5} \\
2.5 \times 10^{5} \\
1.5 \times 10^{9} \\
5 \times 10^{7} \\
5 \times 10^{8}
\end{array}
$$

G3Pmutants $(\%)$

* The letters refer to the points marked in Fig. 3.

Since fosfomycin may select for transport-impaired mutants, the occurrence of such mutants was investigated among the recovered cells. E. coli 185 (pOU900) cultures were subjected to transient lysis by 0.1 and $1 \mathrm{mg}$ fosfomycin $\mathrm{ml}^{-1}$ (final concentration) in $\mathrm{L}$ broth. Then, we assayed surviving cells through the lytic phase for the $\mathrm{G}^{3} \mathrm{P}^{-}$phenotype (Table 1). Whilst treatment with $0.1 \mathrm{mg}$ fosfomycin $\mathrm{ml}^{-1}$ did not modify the phenotype of the surviving population, treatment with $1 \mathrm{mg}$ fosfomycin $\mathrm{ml}^{-1}$ resulted in a progressive enrichment of $\mathrm{G}^{-} \mathrm{P}^{-}$ mutants. Most of these mutants showed glycerol ${ }^{+}$and maltose ${ }^{+}$phenotypes, indicating that they probably carried mutations in the $g l p T$ gene. 
When cells recovered from the treatment with $0 \cdot 1 \mathrm{mg}$ fosfomycin $\mathrm{ml}^{-1}$ were challenged again with the drug they reproduced the lytic phase. However, those cells that survived $1 \mathrm{mg}$ fosfomycin $\mathrm{ml}^{-1}$ were resistant to further lysis by fosfomycin.

\section{DISCUSSION}

Fosfomycin-resistance plasmids have been reported to confer very high levels of resistance to fosfomycin ( $\mathrm{MIC}=60 \mathrm{mg} \mathrm{ml}^{-1}$ ) upon E. coli strains. However, we have shown here that this was probably not the case. E. coli cells carrying fosfomycin-resistance plasmids lyse in the presence of low doses (relative to its MIC) of fosfomycin, and then resume growth up to culture saturation. Since the MIC is determined $16-24 \mathrm{~h}$ after inoculation, it does not reveal what happens in the meantime.

One of the aims of this work was to determine the exact contribution of fosfomycin-resistance plasmids to the resistance of $E$. coli cells carrying them. We have previously observed that the fos gene of fosfomycin-resistance plasmids codes for the modification of intracellular fosfomycin (León et al., 1985). However, this mechanism was not very effective in protecting $E$. coli cells against lysis by fosfomycin. Since we have also shown that $E$. coli cells containing fosfomycinresistance plasmids transport fosfomycin (León et al., 1982). we have interpreted the transient lysis of these cells as a saturation of the system that modifies fosfomycin. This occurred when the transport of fosfomycin was very efficient, as observed when bacteria were grown in rich media. Once the modification system is saturated, fosfomycin accumulates inside the cells, and when it reaches a critical level the cells lyse. On the other hand, cells growing in minimal media did not lyse. This has been interpreted as a consequence of decreased transport of fosfomycin by $E$. coli cells when grown in minimal media. We have ruled out the possibility that this is due to an effect of phosphate concentration in the medium, by using a low phosphate medium (GarenLevinthal) in addition to M9. The systems used to transport fosfomycin into $E$. coli are inducible (Kahan et al., 1974). It is possible that these transport systems would not be induced in cells growing in minimal media, which would therefore result in decreased transport of fosfomycin. The existence of transient lysis means that not all the cells in a culture were lysed by fosfomycin. A small fraction of the cells $\left(1\right.$ in $\left.10^{4}\right)$ remained viable and supported a new phase of growth. A similar phenomenon has been previously described for $E$. coli cultures subjected to the action of cell-wall-active antibiotics, such as penicillin, but this case differs slightly in that the surviving cells do not resume growth unless the antibiotic is removed from the culture medium.

Two types of survivor should be distinguishable after lysis of plasmid-containing cells : (i) cells recovering from lysis by high concentrations of fosfomycin $\left(1 \mathrm{mg} \mathrm{ml}^{-1}\right)$, which were mainly mutants $(90 \%)$ showing impaired G3P transport, and (ii) cells recovering from low doses of fosfomycin, which were not transport mutants and whose recovery was probably due to the resistance mechanism provided by the plasmid.

When E. coli cells containing fosfomycin-resistance plasmids were grown on solid media containing fosfomycin, the appearance of the colonies was delayed, relative to that on antibiotic free medium. In addition, the colonies were translucent in the first $12-16 \mathrm{~h}$ of growth and then adopted their normal opaque character after about $24 \mathrm{~h}$. Cells in these colonies were not transport mutants. These observations suggested that growth on solid media was also affected by a similar transient lytic phase, and that survivors resumed growth through a form resistant to the action of fosfomycin (L forms?) which had translucent colonies before acquiring a normal appearance.

Our conclusion is that the high level of resistance to fosfomycin cannot be explained by the plasmid-determined mechanism alone, but that it results from a combination of two different mechanisms. Higher resistance levels were obtained when a transport defective mutant such as $E$. coli $185 \mathrm{GP}\left(\mathrm{MIC}=1 \mathrm{mg} \mathrm{ml}^{-1}\right.$ ) harboured a fosfomycin-resistance plasmid. This strain, $E$. coli $185 \mathrm{GP}$ (pOU900), was not lysed by any concentration of fosfomycin lower than its MIC (80

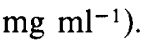


The level of resistance due to the fosfomycin-resistance plasmids alone could be considered as either the concentration which allows continuous growth without transient lysis (about $50 \mu \mathrm{g} \mathrm{ml}^{-1}$ ), or the concentration which did not select for transport defective mutants (about $250 \mu \mathrm{g} \mathrm{ml}^{-1}$ ).

This study was supported by a grant from the Comisión Asesora de Investigación Científica y Técnica.

\section{REFERENCES}

AlPER, M. D. \& AMES, B. N. (1978). Transport of antibiotics and metabolite analogs by systems under cyclic AMP control: positive selection of Salmonella typhimurium cya and crp mutants. Journal of Bacteriology 128, 785-793.

Cordaro, J. C., Melton, T., Stratis, J. P., Atagun, M., Gladding, C., Hartman, P. E. \& Roseman, S. (1976). Fosfomycin resistance: selection method for internal and extended deletions of the phosphoenolpyruvate:sugar phosphotransferase genes of Salmonella typhimurium. Journal of Bacteriology 128 , 785-793.

Davis, R. W., Botstein, D. \& Roth, J. (1980). Advanced Bacterial Genetics. Cold Spring Harbor, NY: Cold Spring Harbor Laboratory.

García-Lobo, J. M. \& OrTiz, J. M. (1982). Tn2921, a transposon encoding fosfomycin resistance. Journal of Bacteriology 151, 457-459.

García-Lobo, J. M., León, J., Navas, J. \& Ortiz, J. M. (1984). Cloning and expression in minicells of the determinant of resistance to fosfomycin from the transposon Tn2921. Plasmid 11, 243-247.

Garen, A. \& Levinthal, C. (1960). A fine structure genetic and chemical study of the enzyme alkaline phosphatase of Escherichia coli. Biochimica et biophysica acta 38, 470-483.

Kahan, F. M., Kahan, J. S., Cassidy, P. J. \& Kropp, H. (1974). The mechanism of action of fosfomycin (phosphonomycin). Annals of the New York Academy of Sciences 235, 364-385.

LEón, J. (1983). Estudio de la resistencia plasmidica a fosfomycina. PhD thesis, Universidad Complutense, Madrid, Spain.

León, J., García-Lobo, J. M., Navas, J. \& Ortiz, J. M. (1982). Fosfomycin resistance plasmids do not affect fosfomycin transport into E. coli. Antimicrobial Agents and Chemotherapy 21, 608-612.

León, J., García-Lobo, J. M., Navas, J. \& Ortiz, J. M. (1985). Fosfomycin-resistance plasmids determine an intracellular modification of fosfomycin. Journal of General Microbiology 131, 1649-1655.

LiN, E. J. J. (1976). Glycerol dissimilation and its regulation in bacteria. Annual Review of Microbiology 30, $535-578$.

LudTke, D., Larson, T. J., Beck, C. \& Boos, W. (1982). Only one gene is required for the $g l p T$ dependent $s n$-glycerol-3-phosphate transport in $E$. coli. Molecular and General Genetics 186, 540-547.

Mendoza, C., Garcia, J. M., Llaneza, J., Mendez, F. J., Hardison, C. \& Ortiz, J. M. (1980). Plasmiddetermined resistance to fosfomycin in Serratia marcescens. Antimicrobial Agents and Chemotherapy 18, 215-219.

TsuruOKA, T. \& YAMADA, Y. (1975). Characterization of spontaneous fosfomycin resistant cells of Escherichia coli B in vitro. Journal of Antibiotics 28, 906-911. 